\title{
Comparative sequence analyses reveal sites of ancestral chromosomal fusions in the Indian muntjac genome Vicky Tsipouri*, Mary G Schueler ${ }^{*}$, Sufen $\mathrm{Hu}^{\dagger}$, NISC Comparative Sequencing Program ${ }^{*}$, Amalia Dutra§, Evgenia Pak§, Harold Riethman ${ }^{\dagger}$ and Eric D Green ${ }^{*}$
}

\begin{abstract}
Addresses: *Genome Technology Branch, National Human Genome Research Institute, National Institutes of Health, 50 South Dr., Bethesda, Maryland, 20892, USA. ${ }^{\dagger}$ Molecular and Cellular Oncogenesis, Wistar Institute, 3601 Spruce Street, Philadelphia, Pennsylvania, 19104, USA. ${ }^{*}$ NIH Intramural Sequencing Center (NISC), 5625 Fishers Ln., Rockville, Maryland, 20852, USA. §Genetic Disease Research Branch, National Human Genome Research Institute, National Institutes of Health, 49 Convent Dr., Bethesda, Maryland, 20892, USA.
\end{abstract}

Correspondence: Eric D Green. Email: egreen@nhgri.nih.gov

Published: 28 October 2008

Genome Biology 2008, 9:RI55 (doi:I0.I I86/gb-2008-9-10-r I55)

The electronic version of this article is the complete one and can be found online at http://genomebiology.com/2008/9/I0/RI55
Received: 29 July 2008

Revised: 15 October 2008

Accepted: 28 October 2008

(c) 2008 Tsipouri et al.; licensee BioMed Central Ltd.

This is an open access article distributed under the terms of the Creative Commons Attribution License (http://creativecommons.org/licenses/by/2.0), which permits unrestricted use, distribution, and reproduction in any medium, provided the original work is properly cited.

\begin{abstract}
Background: Indian muntjac (Muntiacus muntjak vaginalis) has an extreme mammalian karyotype, with only six and seven chromosomes in the female and male, respectively. Chinese muntjac (Muntiacus reevesi) has a more typical mammalian karyotype, with 46 chromosomes in both sexes. Despite this disparity, the two muntjac species are morphologically similar and can even interbreed to produce viable (albeit sterile) offspring. Previous studies have suggested that a series of telocentric chromosome fusion events involving telomeric and/or satellite repeats led to the extant Indian muntjac karyotype.
\end{abstract}

Results: We used a comparative mapping and sequencing approach to characterize the sites of ancestral chromosomal fusions in the Indian muntjac genome. Specifically, we screened an Indian muntjac bacterial artificial-chromosome library with a telomere repeat-specific probe. Isolated clones found by fluorescence in situ hybridization to map to interstitial regions on Indian muntjac chromosomes were further characterized, with a subset then subjected to shotgun sequencing. Subsequently, we isolated and sequenced overlapping clones extending from the ends of some of these initial clones; we also generated orthologous sequence from isolated Chinese muntjac clones. The generated Indian muntjac sequence has been analyzed for the juxtaposition of telomeric and satellite repeats and for synteny relationships relative to other mammalian genomes, including the Chinese muntjac.

Conclusions: The generated sequence data and comparative analyses provide a detailed genomic context for seven ancestral chromosome fusion sites in the Indian muntjac genome, which further supports the telocentric fusion model for the events leading to the unusual karyotypic differences among muntjac species. 


\section{Background}

The number of chromosomes in the mammalian nuclear genome is generally well-confined, typically ranging from 36 to 60. There are, however, rare exceptions. At one extreme, the genome of the red viscacha rat (Tympanoctomys barrerae) consists of 102 chromosomes [1]; at the other extreme, that of the Indian muntjac (Muntiacus muntjak vaginalis) consists of a modest 6 and 7 chromosomes (in the female and male, respectively) [2]. Understanding the molecular basis for such radically different mammalian karyotypes would provide insight about the evolutionary history that has led to architecturally distinct genomes. Furthermore, comparative studies of mammalian karyotypes can more generally advance our understanding of vertebrate genome evolution [3].

Muntjacs belong to the suborder Ruminantia, which includes Moschidae (musk deer), Tragulidae (chevrotains and mouse deer), Antilocapridae (pronghorns), Giraffidae (giraffes and okapis), Bovidae (cattle, sheep, goats, and antelopes), and Cervidae (deer). The Cervidae family includes moose, caribou, deer, and muntjacs, with the various species inhabiting Europe, Asia, North Africa, and the Americas [4]. Muntjacs have interested cytogeneticists and genomicists because of the markedly different karyotypes that are present in closely related species [5]. In contrast to the strikingly low chromosome number in the Indian muntjac, its close relative - the Chinese muntjac (Muntiacus reevesi) - has a chromosome number that is more typical of a mammal, with 46 in both sexes [6]. The total genome size in Chinese and Indian muntjacs is believed to differ only slightly, with haploid C-values of 2.7 and $2.1 \mathrm{pg}$, respectively [7]; as such, the physical chromosome lengths vary tremendously between the species. Indian and Chinese Muntjacs are morphologically similar and can interbreed to produce viable (although sterile) offspring [8]. Interestingly, there are muntjac species with genomes with intermediate numbers of chromosomes: Muntiacus feae has 13 and 14 chromosomes in the female [9] and male [10], respectively, while both Muntiacus crinifrons and Muntiacus gongshanensis have 8 and 9 chromosomes in the female and male, respectively [11]. In general, muntjacs are thought to have been subjected to the fastest rate of evolutionary change with respect to chromosome number among the vertebrate lineages [10].

Various studies have investigated the molecular and evolutionary events that have yielded the highly unusual Indian muntjac karyotype. Emerging from those studies is the hypothesis that tandem chromosome fusion events occurred during the evolution of the muntjac lineage, resulting in the small number of large chromosomes seen in the modern-day Indian muntjac [12,13]. Molecular cytogenetic studies have provided the most compelling evidence for such a tandem chromosome fusion hypothesis. These have demonstrated the presence of centromeric satellite repeats [14] and telomeric repeats [15] at interstitial positions of Indian muntjac chromosomes by fluorescence in situ hybridization (FISH) and established Indian muntjac-human comparative genome maps by chromosome painting [16,17]. Comparative FISH studies using chromosome-specific probes derived from flowsorted muntjac chromosomes have further suggested that the extant Indian muntjac karyotype was derived from an ancestral deer karyotype with a diploid genome of 70 chromosomes that underwent a series of chromosome fusion events and other chromosomal rearrangements [18]; several putative junction regions (that is, genomic sites where ancestral chromosomes have fused together) were identified in these studies.

More recently, four telomeric-satellite I repeat junctions on Indian muntjac chromosomes were sequenced $[19,20]$, and mapping studies with bacterial artificial chromosomes (BACs) helped to define the orientation of other putative ancestral chromosome fusion sites in the Indian muntjac genome [21]. Specifically, evidence for centromere-telomere (head-to-tail) fusions was encountered in the arms of Indian muntjac chromosomes, whereas that for centromere-centromere (head-head) fusions was found at the centromeres. The 46-chromosome karyotype of the Chinese muntjac is thought to have evolved from a common 70-chromosome ancestral species through 12 tandem fusions involving 18 chromosomes [22]. Additional studies using cross-species BAC mapping suggested that the tandem fusions that occurred during the karyotypic evolution of the closely related species Muntiacus crinifrons, M. feae, and M. gongshanensis also had a centromere-telomere (head-to-tail) orientation [23,24].

The above model for muntjac chromosome evolution involves a number of known repetitive sequences that are associated with either telomeres or centromeres. For example, as with certain other mammals, muntjacs harbor the repeat (TTAGGG) $_{\mathrm{n}}$ at their telomeres [15]. In some mammals (for example, human), this repeat unit is also found intrachromosomally in various forms: subtelomerically (including degenerate instances), as head-to-head (that is, telomere-totelomere) fusion products [25], and within short, essentially exact repeat stretches [26]. Also implicated in the above model are Cervidae-specific centromeric satellite sequences, including satellites I, II, and IV. Satellite I (MMVsatIA in Indian muntjac [27], $\mathrm{C}_{5}$ in Chinese muntjac [14]) is roughly 1 $\mathrm{kb}$ in length and contains internal 31 bp subrepeats $[28,29]$. Satellite II (Mmv-0.7 in Indian muntjac [30]) is $0.9 \mathrm{~kb}$ in length. Immunoprecipitation of Indian muntjac DNA with human anti-centromere autoantibodies has shown that satellite II associates with centromeric protein A (CENPA) [31] and participates in the formation of the muntjac kinetochore. Satellite IV (MMV-1.o in Indian muntjac, MR-1.o in Chinese muntjac) is roughly $1 \mathrm{~kb}$ in length and is highly similar to satellite II [32]. Characterization of these three satellite sequences in the Formosan muntjac (Muntiacus reevesi micrurus), a subspecies of Chinese muntjac with the same number of chromosomes, revealed the following 
chromosomal organization: pter - II - IV - I - qter [33]. Additionally, satellite II (FM-satII in Formosan muntjac) co-localizes with telomeric sequences on Formosan muntjac chromosomes [33], indicating that Formosan (and likely Chinese) muntjac chromosomes are truly telocentric.

We sought to use comparative sequencing to establish a more detailed view of the evolutionary events leading to the unusual Indian muntjac karyotype. Here, we report the sequencing and characterization of a number of genomic regions in the Indian muntjac genome that represent sites of ancestral chromosome fusion events. Comparative analyses of the generated sequences with the orthologous genomic regions in the Chinese muntjac and several other mammalian species reveal details about the molecular architecture and the likely evolutionary history of the Indian muntjac genome.

\section{Results \\ BAC isolation, mapping, and characterization}

We reasoned that Indian muntjac BACs containing regions corresponding to ancestral chromosome fusion sites would likely contain remnant telomeric-repeat sequences. We thus screened an approximately 11-fold redundant Indian muntjac BAC library with probes specific for telomeric repeats, and found that a large number of clones (approximately 3,000 or approximately $1.4 \%$ of the library) yielded at least a weak hybridization signal. The 343 BACs with the strongest hybridization signals were selected for further study. Restriction enzyme digest-based fingerprint analysis [34] allowed the selected clones to be assembled in 45 multi-clone contigs. Southern blot analysis of the BACs in the 19 largest contigs (each containing at least 4 clones and averaging 6.9 clones) confirmed the presence of telomeric-repeat sequences in all clones, and these repeat sequences consistently resided on the same-sized restriction fragment in all BACs from a given contig.

Southern blot analysis was also performed using probes designed from previously reported muntjac satellite-telomeric repeat junctions [19]. BACs from 7 of the 19 largest contigs were found to hybridize to at least 1 of these probes; representative BACs from each of these 7 contigs were studied further by FISH. The presence of various repeats (for example, telomeric- and centromeric-repeat sequences) in the isolated BACs typically resulted in complex FISH patterns, as illustrated Figure 1. Strong hybridization signals were observed at the euchromatin/heterochromatin boundary of the $\mathrm{X}$ centromere. These hybridization results are similar to those obtained with other probes containing satellite I (for example, $\mathrm{C}_{5}$ and TGS400) [14,19]. With this initial set of clones, we at best encountered BACs hybridizing strongly to only two locations in the Indian muntjac genome (Figures 1a,b); typically, the analyzed clones hybridized to many more sites (Figure 1c).

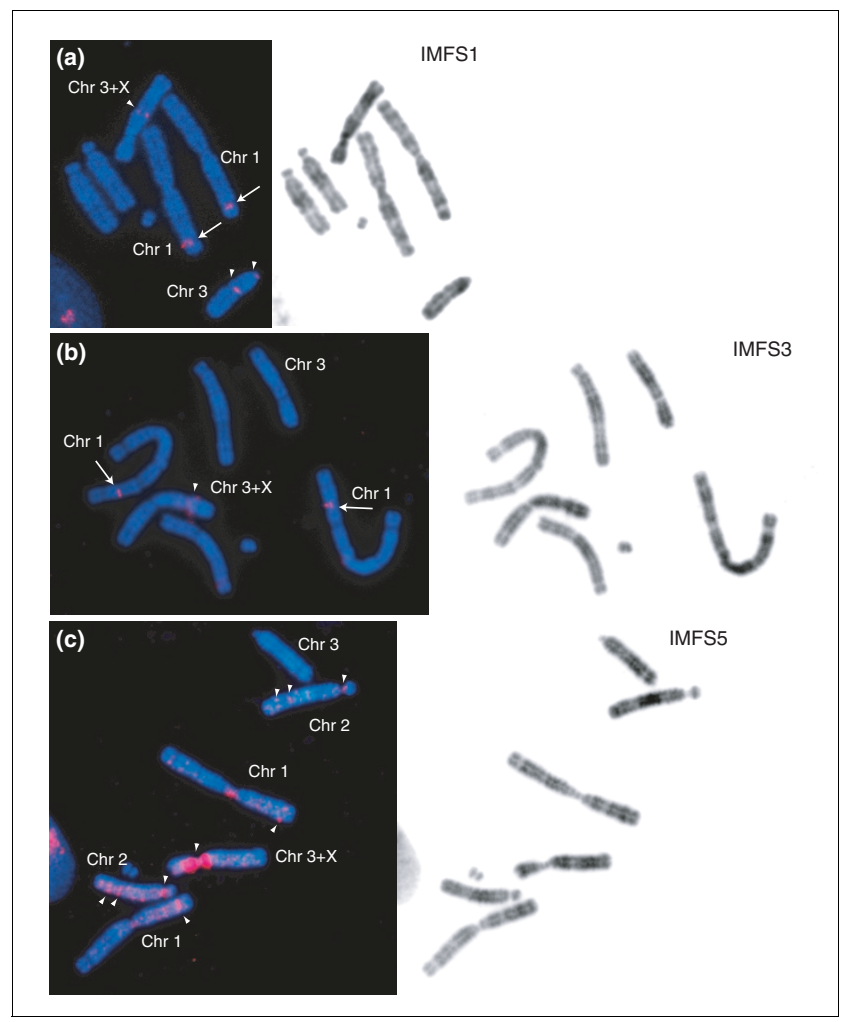

Figure I

FISH mapping of Indian muntjac BACs. Three Indian muntjac BACs (whose sequences correspond to accession numbers (a) [GenBank:ACI54I46], (b) [GenBank:ACI52355], and (c) [GenBank:ACI54920]) were mapped by FISH to metaphase spreads prepared from an Indian muntjac fibroblast cell line. Hybridization is seen at: (a) interstitial positions on chromosomes I (arrows), 3, and $3+X$, as well as the centromere of chromosome 3 (the signals on 3 and $3+X$ are indicated with arrowheads); (b) an interstitial position on chromosome I (arrows) and at the neck of chromosome $3+X$ (arrowhead); and (c) multiple sites on various Indian muntjac chromosomes (arrowheads). FISH composite images generated from merging the DAPI (blue) and Spectrum Orange (red) channels (left) and inverted DAPI banding images (right) are provided in each case. Based on further studies (see text for details), the origins of the analyzed BACs were ultimately found to be on chromosome $I$ in the case of $(a, b)$, but not yet established in the case of (c); further, the analyzed clones were found to contain the indicated ancestral chromosome fusion sites (IMFSI, IMFS3, and IMFS5, respectively; Table I).

\section{Generation and assimilation of Indian and Chinese muntjac genomic sequences}

Based on our BAC mapping and characterization studies, we selected and sequenced [35] one clone from each of the above seven Indian muntjac BAC contigs. We also sequenced a small number of Indian muntjac BACs ([Gen-

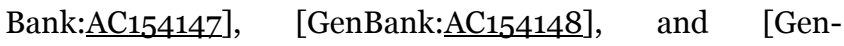
Bank:AC154923]; data not shown) that appeared to contain telomeric repeats based on hybridization studies, but were not positive with any of the probes designed from previously reported muntjac satellite-telomeric repeat junctions. Subsequently, we isolated and sequenced additional overlapping BACs to extend the sequence coverage of these regions. In 
Table I

Generated sequences of Indian muntjac chromosome fusion sites

\begin{tabular}{|c|c|c|c|c|c|c|}
\hline Name & Accession numbers* & $\begin{array}{l}\text { Sequence length } \\
\text { (bp) }\end{array}$ & $\begin{array}{c}\text { Number of } \\
\text { sequencing gaps }\end{array}$ & $\begin{array}{c}(\text { TTAGGG })_{n} \text { repeats } \\
(\mathrm{bP})^{\S}\end{array}$ & Total satellite I (bp) & $\begin{array}{l}\text { Indian muntjac } \\
\text { chromosome }\end{array}$ \\
\hline IMFSI & [GenBank:DP000824] & 292,157 & 9 & $367 \#$ & 22,121 & 1 \\
\hline IMFS2 & [GenBank:DP000825] & 209,878 & 3 & 616 & 36,382 & 1 \\
\hline IMFS3 & [GenBank:DP000827] & 460,923 & 7 & $24,25,162,341$ & $105,769 * *$ & 1 \\
\hline IMFS4 & [GenBank:DP000826] & 324,188 & 2 & 22,413 & 12,225 & 3 \\
\hline IMFS5 & [GenBank:DP000830] & 172,824 & 2 & 168 & $72,301 * *$ & Unknown \\
\hline IMFS6 & [GenBank:DP000828] & 248,686 & 2 & $185^{\prime}, 26 \mid \#$ & 61,861 & 2 \\
\hline IMFS7 & [GenBank:DP000829] & $\mathrm{I74,7|I+ \dagger}$ & 2 & 837 & 87,048 & Unknown \\
\hline
\end{tabular}

*Each IMFS sequence was assembled using the generated sequences from two or more BACs (except for IMFS5, which was derived from a single BAC sequence). The indicated GenBank accession numbers correspond to the assembled sequences (also see Figures 3 and 6 ). thength of the assembled IMFS sequence. $¥$ Total number of gaps within the sequences of the individual BACs used to generate the IMFS sequence. Note that there

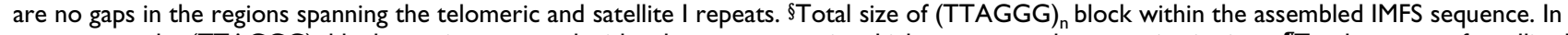
some cases, the (TTAGGG) blocks are interspersed with other sequences, in which case more than one size is given. ITotal amount of satellite I

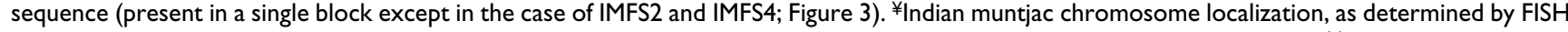
studies of individual BACs (Table 3). \#A similar repeat, (TTCGGG) ${ }_{n}$, resides immediately adjacent to the (TTAGGG) ${ }_{n}$ block. **Satellite I sequence resides in a single block that is interrupted by LI and MER66-int LTR/ERVI repeats. ttThe chromosome fusion site within IMFS7 contains a region of $100 \%$ sequence identity with TGS400 [19].

total, we generated the sequence for 18 BACs (Table 1), yielding approximately $1.88 \mathrm{Mb}$ of Indian muntjac genomic sequence.

We also sequenced six Chinese muntjac BACs (approximately $0.99 \mathrm{Mb}$ total) derived from genomic regions (Chinese muntjac telomere (CMTel)1, CMTel3, CMTel4, and Chinese muntjac satellite (CMSat)4; Table 2) that are orthologous to some of the generated Indian muntjac sequence. In all cases, sequence contigs were ordered and oriented, ensuring that the correct long-range organization of the sequence was established [35]. In some cases, sequence gaps were filled using standard sequence-finishing routines [36]. Each set of overlapping BAC sequences was assembled into a single nonredundant sequence, which in turn was manually verified and submitted to GenBank ([GenBank:DPooo820-DPooo830];
Tables 1 and 2). Each of the resulting seven assembled Indian muntjac genomic sequences are presumed to contain a different ancestral chromosome fusion site (designated IMFS1 to IMFS7 for Indian muntjac fusion site; Table 1). A previously reported junction sequence (TGS400) [19] lies within IMFS7. The other six IMFSs, although of similar repeat composition, do not match any of the previously described junction sequences $[19,20]$, indicating that they represent novel chromosome fusion sites.

\section{Detection of repetitive and duplicated sequences}

Characterization of the generated Indian muntjac sequences involved detection and classification of repetitive sequences. In addition to typical transposable-element repeats (for example, long interspersed nucleotide elements (LINEs), short interspersed nucleotide elements (SINEs), and long ter-

Table 2

Chinese muntjac sequences orthologous to Indian muntjac chromosome fusion sites

\begin{tabular}{|c|c|c|c|c|c|}
\hline Name & Orthologous sequence* & Accession numbers ${ }^{\dagger}$ & Sequence length (bp) $\ddagger$ & Number of sequencing gaps§ & $(\text { TTAGGG) })_{n}$ repeats (bp) \\
\hline CMTell & IMFSI & [GenBank:DP000822] & 202,617 & 4 & None \\
\hline CMTel3 & IMFS3 & [GenBank:DP00082I] & 292,786 & 3 & 24 \\
\hline CMTel4 & IMFS4 & [GenBank:DP000820] & 286,938 & 3 & None \\
\hline CMSat4 & IMFS4 & [GenBank:DP000823] & 215,295 & 5 & None \\
\hline
\end{tabular}

*CMTel reflects Chinese muntjac sequence that is orthologous to the telomeric side of the corresponding IMFS sequence; CMSat reflects Chinese muntjac sequence that is orthologous to the satellite side of the corresponding IMFS sequence. CMTel sequences are not necessarily subtelomeric in the Chinese muntjac genome, but were likely subtelomeric in a shared ancestor with Indian muntjac. Similarly, CMSat sequences are not necessarily pericentromeric in the Chinese muntjac genome, but were likely pericentromeric in a shared ancestor with Indian muntjac (see text). The relationship between IMFS and CMTel/CMSat sequences is shown in Figures 3 and 6. Cytogenetic localization of individual BACs used to generate Chinese muntjac sequences is provided in Table 4. tThe indicated GenBank accession numbers correspond to each sequenced BAC used to generate the assembled Chinese muntjac sequence. ¥Length of the assembled multi-BAC or individual BAC Chinese muntjac sequence. $\S$ Total number of gaps within the sequences of individual BACs used to generate the CMTel/CMSat sequence. ITotal size of (TTAGGG)n block within the assembled sequence. 
minal repeats (LTRs)), we paid particular attention to the presence of known telomeric and centromeric repeats (Figure 2). All seven Indian muntjac sequences listed in Table 1 (IMFS1 through IMFS7) were found to contain at least one

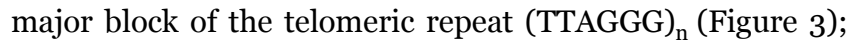
these blocks range from 168 to 837 bases in length. Sequences IMFS4, IMFS6, and IMFS3 have 1, 1, and 3 additional telomeric-repeat blocks, respectively; the additional blocks are shorter than the others ( 22 to 185 bases). In all cases, the individual (TTAGGG) ${ }_{\mathrm{n}}$ monomers are oriented in the same direction; in no case did we encounter a head-to-head configuration $\left(5^{\prime} \text { (TTAGGG) }\right)_{\mathrm{n}}$-(CCCTAA $)_{\mathrm{n}} 3$ '; for example, as found on human chromosome 2q13 [37]). In IMFS1 and IMFS6, a similar repeat - (TTCGGG) - resides immediately adjacent to the more common (TTAGGG) $)_{n}$ telomeric repeat (Figure 3).

Additionally, all seven Indian muntjac sequences were found to contain centromeric satellite I repeat sequences immediately adjacent to the telomeric-repeat block (Figure 3), similar to that found previously in the Indian muntjac genome [19]. The amount of satellite I differs among the sequences, ranging from roughly $12 \mathrm{~kb}$ (IMFS4) to over $105 \mathrm{~kb}$ (IMFS3). The satellite I sequences are rarely interrupted by other repeats; exceptions include the presence of MER66-int long terminal repeat/endogenous retrovirus (LTR/ERV) and L1 repeats that interrupt the satellite I sequences in $\mathrm{IMFS}_{5}$ and $\mathrm{IMFS}_{3}$, respectively. Of note, IMFS1, IMFS4, IMFS 5 , and IMFS7 also contain a small block of centromeric satellite IV sequence [32] (Figure 3). IMSF4 additionally has two short blocks of satellite II on the opposite strand of the satellite IV repeat; satellites II and IV are known to be highly similar [32].

Pericentromeric regions of mammalian chromosomes frequently harbor segments that are present in more than one copy in the genome [38]. These duplicated segments typically originate from various ancestral genomic locations and are physically juxtaposed with centromeric satellites. Copies of each duplicated segment usually have high pair-wise sequence identity due to the relatively recent occurrence of the duplication event.

We analyzed the generated Indian muntjac sequences for the presence of duplicated segments. In all 7 of the chromosome fusion sites characterized here, at least one duplicated segment was found to reside immediately adjacent to satellite I (Figure 3). Further, all duplicated segments depicted in Figure 3 are at least $1 \mathrm{~kb}$ in size (typically much larger) and share 94-98\% pair-wise sequence identity with their matching colored block(s) in Figure 3. Of note, the duplicated segment present in IMFS2 and IMFS4 (light beige block in Figure 3) is an exception, having only $82 \%$ sequence identity between copies. IMFS 1 has a large ( $>60 \mathrm{~kb}$; reddish brown block in Figure 3) duplicated segment that is also present in IMFS2; within this duplicated segment are 5 regions that are over $3 \mathrm{~kb}$ in size, which share $95-98 \%$ sequence identity (3 of these regions are over $11 \mathrm{~kb}$ in size), and which reside in the same relative order and orientation in IMFS1 and IMFS2. An approximately $10 \mathrm{~kb}$ duplicated segment (brown block in Figure 3 ) is present in 5 other chromosome fusion sites (IMFS3IMFS7), with 94-97\% pair-wise sequence identities. IMFS1, IMFS4, and IMFS6 share another duplicated segment that is greater than $10 \mathrm{~kb}$ in size and has $96-98 \%$ sequence identify among copies (beige block in Figure 3). Different combinations and spatial arrangements of these duplicated segments are seen among the seven chromosome fusion sites (Figure 3).

Similar analyses were performed with the generated orthologous Chinese muntjac sequences. As with the Indian muntjac sequences, generic classes of repeats (for example, LINES, SINES, and LTRs) were identified. Additionally, a short block of (TTAGGG) repeats (24 bp) was found in CMTel3; no centromeric satellites were found in any of the Chinese muntjac sequences. Consistent with the Chinese muntjac sequences being of telomeric and not centromeric origin, none contain duplicated segments (based on comparisons with each other and with their orthologous Indian muntjac sequences; Figure 3). This result was expected given that the Chinese muntjac BACs were selected to be orthologous to the non-repetitive regions of IMFSs.

\section{Synteny analysis and gene annotation}

We performed a systematic analysis to establish the synteny relationships of the IMFS sequences relative to the human, cow, dog, and mouse genomes. For all seven IMFS sequences, the regions immediately flanking the putative ancestral chromosome fusion site were found to be orthologous to a different chromosome in all of the other species, indicating a breakage of synteny (Figure 4). For example, in the case of IMFS1: the telomeric repeat-containing side is orthologous to human chromosome 8q24.12, cow chromosome 14, dog chromosome 13, and mouse chromosome 15; and the satellite repeat-containing side contains two duplicated segments that are orthologous to human chromosomes 2q33.3 and 1q24.1, cow chromosomes 2 and 3 , dog chromosomes 37 and 38, and mouse chromosome 1 . There is no evidence for synteny breaks in the orthologous regions of the human, cow, dog, or mouse genomes, suggesting that the unique features of the muntjac genome are the result of relatively recent events.

Comparison of the generated Indian muntjac sequences with the human, dog, and cow genome sequences revealed the presence of a number of annotated genes (Table 3). In some instances, the duplicated segments confounded this analysis (for example, sequences matching the gene FLJ40432 reside in both IMFS1 and IMFS2, and those matching BX538248 reside in both IMFS2 and IMFS4). When available, the orthologous Chinese muntjac sequence consistently showed 
(a)
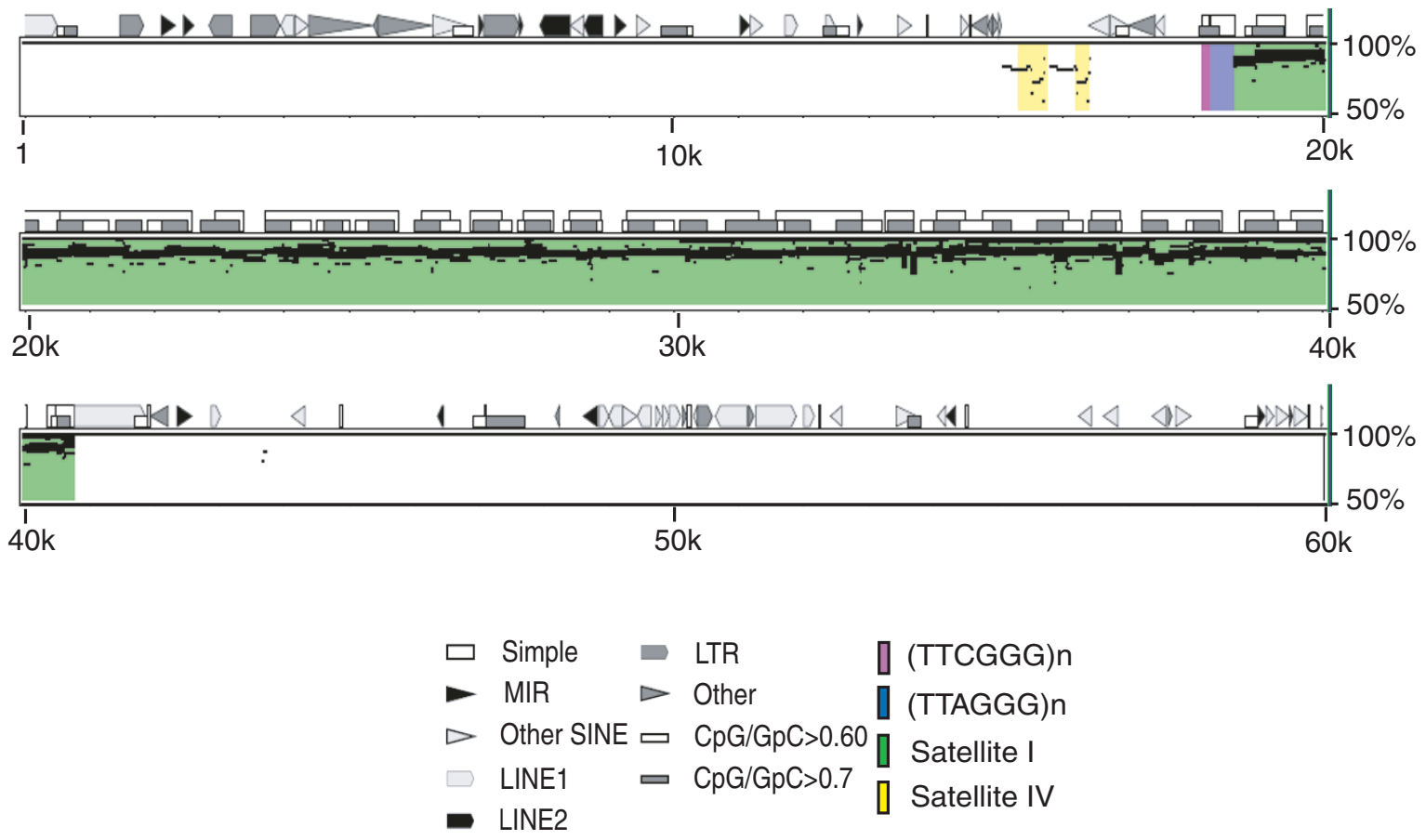

(b)

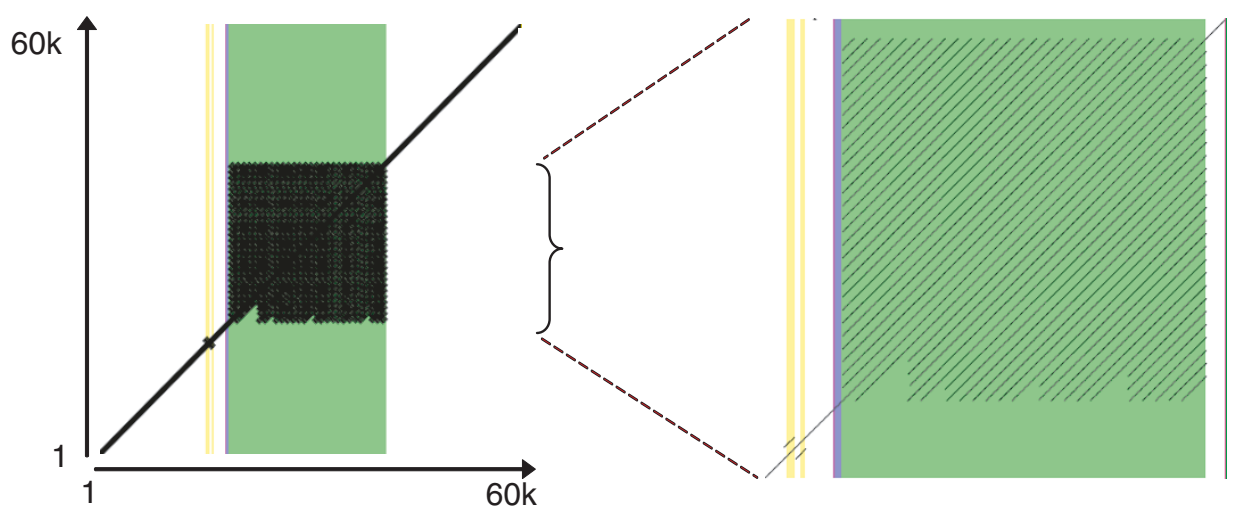

प(TTCGGG)n 【(TTAGGG)n

Uatellite I

प Satellite IV

\section{Figure 2}

Self-self comparative sequence analysis of an Indian muntjac chromosome fusion site. A $60 \mathrm{~kb}$ sequence within IMFSI was compared to itself using PipMaker [63]. (a) Pip plot reveals the putative chromosome fusion site, which consists of a stretch of telomeric repeats (TTAGGG) (blue), and then a large segment of centromeric satellite I (green); note that the latter has extensive amounts of self-self aligning sequences (reflecting satellite I monomers with high sequence identity). Also highlighted are additional features of interest: satellite IV (yellow) and a short stretch of (TTCGGG) (purple). (b) Dot plot of the same $60 \mathrm{~kb}$ region shown in (a). Expanded view reveals the periodic nature of the satellite I monomers.

the same gene order and orientation as the Indian muntjac sequence (Table 4). The presence of conserved gene order within the orthologous sequences on the telomeric side of the fusion site supports the reported orthologous relationships.
The juxtaposition of these gene-containing sequences with centromeric satellites and pericentromeric elements indicates a breakage of synteny relative to all of the other genomes being compared. 
IMFS1

IMFS2

IMFS3

IMFS4

IMFS5

IMFS6

IMFS7
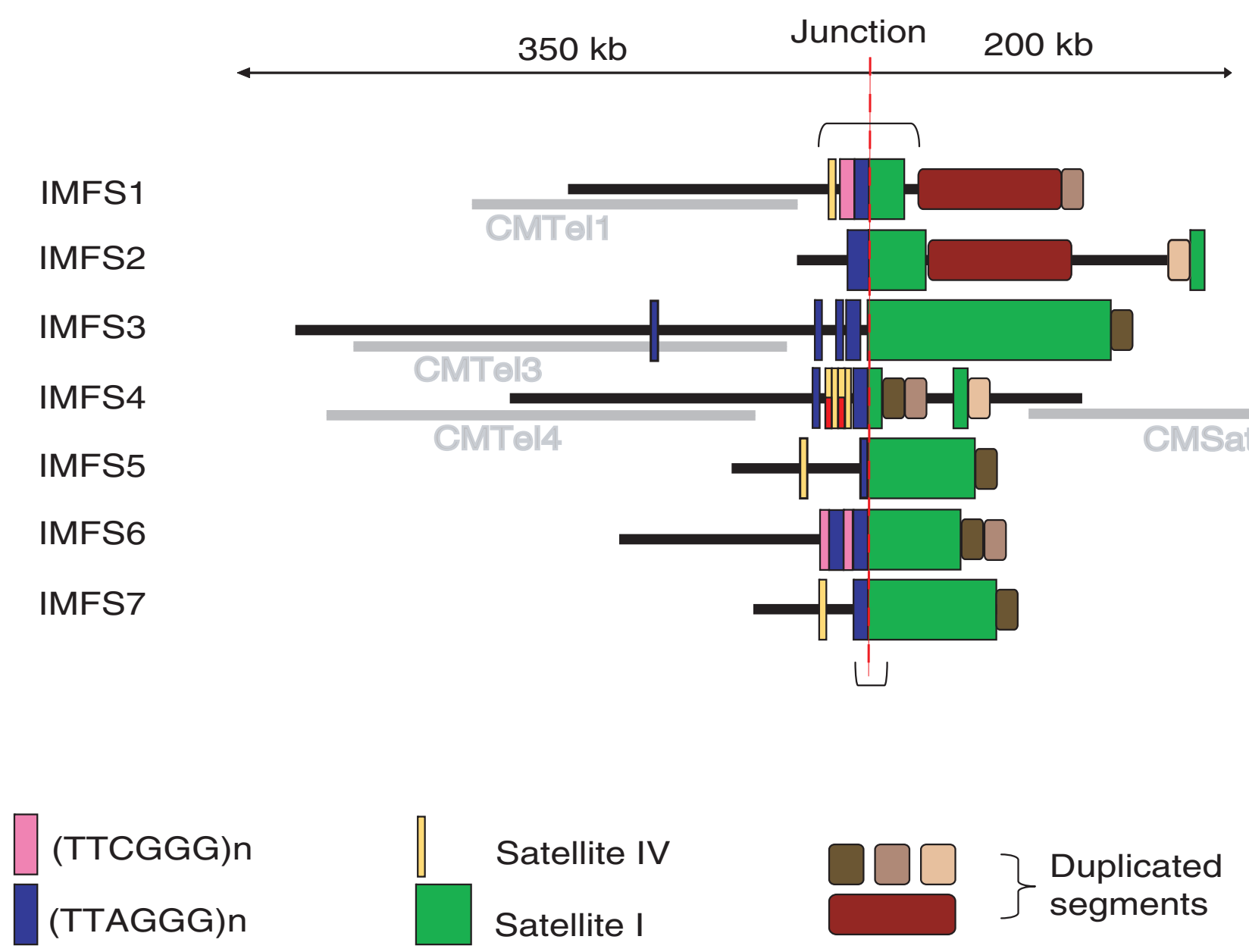

\section{Satellite II}

\section{Figure 3}

Long-range organization of chromosome fusion sites in Indian muntjac. The content and organization of the seven generated Indian muntjac sequences (black lines) is depicted. The positions of (TTAGGG) (blue), (TTCGGG) $_{n}$ (purple), satellite I (green), and satellite IV (yellow) blocks as well as duplicated segments (brown and beige) are indicated. Generated orthologous Chinese muntjac sequences are shown in gray (for IMFSI, IMFS3, and IMFS4 only). The junction is defined as the point where the (TTAGGG) ${ }_{n}$ telomeric repeats are fused with satellite I repeats (red dashed line). The bracketed area of IMFSI indicates the region depicted in Figure 2; the bracketed area of IMFS7 indicates the region matching TGS400 [19].

\section{Genomic locations of Indian muntjac chromosome fusion sites}

We sought to establish the genomic locations of the generated Indian muntjac sequences and to trace their evolutionary history relative to the Chinese muntjac genome. IMFS3, the largest generated sequence (spanning $>450 \mathrm{~kb}$; Table 1 ), contains the expected features of an ancestral chromosome fusion site: a telomeric-repeat block, satellite I [14], duplicated segments, and a novel breakage of synteny (Figures 3 and 4). IMFS3 was

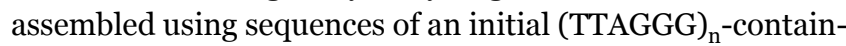
ing BAC [GenBank:AC152355] and two overlapping clones ([GenBank:AC197641] and [GenBank:AC166188]; Figure 5a). FISH studies revealed that BAC [GenBank:AC152355] mapped to Indian muntjac chromosome 1 (Figure 1b), while
BACs ([GenBank:AC197641] and [GenBank:AC166188]; Figure $5 \mathrm{~b}$ ) both hybridized to a pair of interstitial sites on chromosome 1. BAC [GenBank:AC166188] also hybridized to various centromeres and other interstitial sites, likely due to the presence of satellite I and duplicated segments.

We were able to generate Chinese muntjac sequence orthologous to IMFS3. Two overlapping Chinese muntjac BACs (isolated with a probe derived from Indian muntjac BAC [GenBank:AC152355]) were sequenced, resulting in contig CMTel3 (Table 2). FISH studies revealed that both clones ([GenBank:AC196603] and [GenBank:AC198815]) co-localize to a pair of Chinese muntjac telomeres. Sequences on the telomeric side of $\mathrm{IMFS}_{3}$ as well as $\mathrm{CMTel}_{3}$ sequences are 


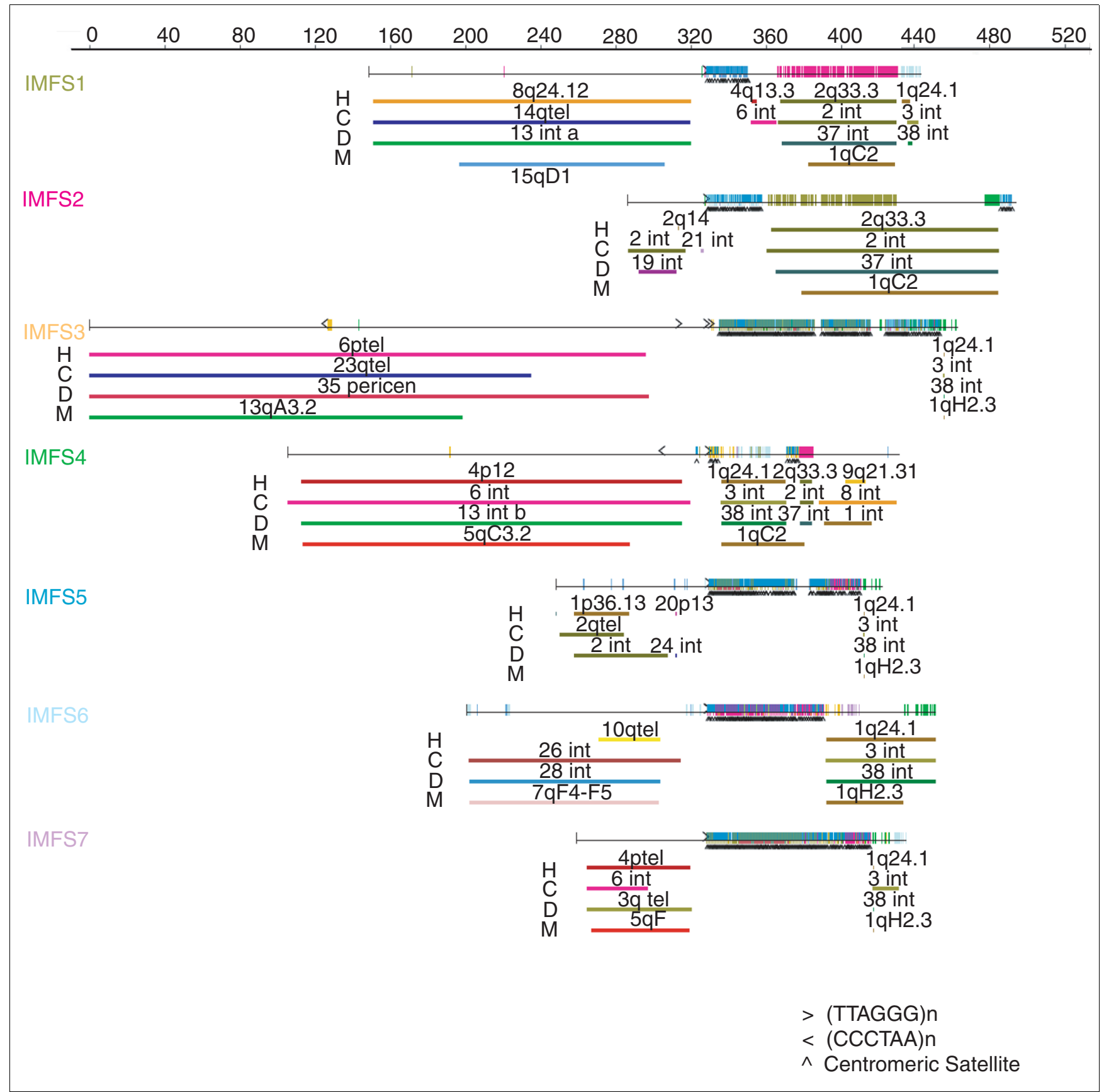

\section{Figure 4}

Synteny relationships between IMFS sequences and corresponding human, cow, dog, and mouse genome sequences. Each generated Indian muntjac sequence (IMFSI-IMFS7) is depicted and represented by a different color; the vertical hatch marks on each sequence indicate identity with other IMFS sequences and are colored to correspond with those IMFS sequences. Telomere (TTAGGG) repeats are indicated with black arrows per their orientation; also indicated are centromeric satellite sequences (both with a black caret symbol and shaded blue). Tracks below each IMFS depict regions of synteny with the indicated genome $(\mathrm{H}$, human; $\mathrm{C}$, cow; $\mathrm{D}$, dog; and $\mathrm{M}$, mouse) as determined by BLAST-based alignments, with the chromosome location in the respective genome indicated in each case.

orthologous to the telomeric region of human chromosome 6p25.3 and cow chromosome 23qtel. These sequences contain genes Dusp22, Irf4, and Exoc2 (Tables 3 and 4). Based on the known synteny relationships among the human, cow, and Chinese muntjac genomes [39], we can deduce that
CMTel3 maps to chromosome 22 in the Chinese muntjac genome. Thus, IMFS3 appears to contain the fusion site between the telomere of an ancestral chromosome related to Chinese muntjac chromosome 22 and, based on the comparative chromosome map [21] and the presence of satellite I and 
Table 3

\begin{tabular}{|c|c|c|c|c|}
\hline Name & Telomeric side* & Functiont & Centromeric side $\ddagger$ & Functiont \\
\hline \multirow[t]{3}{*}{ IMFSI } & Syntrophin betal, component & Cytoskeleton & 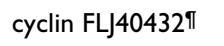 & Cell cycle \\
\hline & Sntbl & & AK024850币 & Part of frizzled 5 \\
\hline & & & Frizzled 5, Fzd5 & Signal transduction \\
\hline \multirow[t]{3}{*}{ IMFS2 } & None & NA & cyclin FLJ40432 & Cell cycle \\
\hline & & & AK0248505 & Part of frizzled 5 \\
\hline & & & BX538248 & Unknown \\
\hline \multirow[t]{5}{*}{ IMFS3 } & FLJ $40227 \S$ & Unknown & None & NA \\
\hline & AKI2575I§ & Unknown & & \\
\hline & Dual specificity protease $22, \mathrm{Dsp} 22$ & $\begin{array}{l}\text { Protein tyrosine/serine/threonine phosphatase } \\
\text { activity }\end{array}$ & & \\
\hline & Interferon regulatory factor 4 , Irf4 & $\begin{array}{l}\text { Transcriptional activator (multiple myeloma } \\
\text { oncogene I) }\end{array}$ & & \\
\hline & Exocyst complex component 2, Exoc2 & Transport & & \\
\hline \multirow[t]{5}{*}{ IMFS4 } & $\begin{array}{l}\text { Nuclear transcription factor, X-box binding-like, } \\
\text { Nfxll }\end{array}$ & Transcription factor & BX538248 & Unknown \\
\hline & Cyclic nucleotide gated channel alpha I, Cnga I & Potassium ion transport & & \\
\hline & NIPA-like domain containing I, Npall & Unknown & & \\
\hline & Tyrosine kinase, $T x k$ & Tyrosine protein kinase, transcription factor & & \\
\hline & Tyrosine-protein kinease, $T e c$ & Tyrosine protein kinase, signaling & & \\
\hline IMFS5 & None & NA & None & NA \\
\hline \multirow[t]{2}{*}{ IMFS6 } & ZNF cluster & Transcription factors & None & NA \\
\hline & Cytochrome P450 2EI, Cyp2el & Metabolism & & \\
\hline IMFS7 & 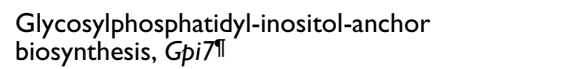 & Biosynthesis & None & NA \\
\hline
\end{tabular}

*Proximal side of telomeric-satellite I junction (Figure 3). tThe functionality of these genes has not been verified; table could include pseudogenes and gene fragments. $\neq$ Distal side of the telomeric-satellite I junction (Figure 3), including duplicated segments. \$Predicted gene (UCSC Genome Browser [7I]). TProvisional gene (UCSC Genome Browser). NA, not applicable.

duplicated segments, the centromere of an ancestral chromosome related to Chinese muntjac chromosome 12 (Figures 5a and 6).

In a similar fashion, we established that IMFS1 resides on Indian muntjac chromosome 1. IMFS1 includes the assembled sequence derived from the two Indian muntjac BACs, while the orthologous CMTel1 represents the sequence of the Chinese muntjac BAC. Indian muntjac BAC [GenBank:AC189002] and Chinese muntjac BAC [GenBank:AC187414] were isolated with a probe derived from Indian muntjac BAC [GenBank:AC154146] (Figure 1a). FISH studies revealed that both Indian muntjac BACs map to Indian muntjac chromosome 1 , while the Chinese muntjac BAC maps to a pair of Chinese muntjac telomeres. Sequences on the telomeric side of IMFS1 as well as CMTel1 sequences are orthologous to human chromosome 8q24.14 and cow chromosome 14qtel in a genomic region that contains Sntb1 (Tables 3 and 4). Based on the known synteny relationships among the human, cow, and Chinese muntjac genomes [39], we can deduce that CMTel1 maps to chromosome 12 in the Chinese muntjac genome. Thus, IMFS1 appears to contain the fusion site between the telomere of an ancestral chromo- some related to Chinese muntjac chromosome 12 and, based on the comparative chromosome map [21] and the presence of satellite I and duplicated segments, the centromere of an ancestral chromosome related to Chinese muntjac chromosome 3c (Figure 6).

The remaining five Indian muntjac sequences (IMF2, IMF4, IMF5, IMF6, and IMF7) could not be unambiguously matched to predicted chromosome fusion sites [21] based on the available data, as detailed below.

The sequence on the telomeric side of IMFS2 is orthologous to the telomeric region of human chromosome 2q14.3 and cow chromosome 2. Based on the known synteny relationships (see above), we can deduce that the IMFS2 telomeric side maps to Chinese muntjac chromosome 3. Using the same logic as above, IMFS2 thus appears to contain the fusion site between the telomere of an ancestral chromosome related to Chinese muntjac chromosome 3 and the centromere of another ancestral chromosome. Based on the comparative chromosome map [21] and our FISH studies of IMFS2, there are three potential matching fusion sites on Indian muntjac chromosome 1: 3c/3d, 3b/17, and 3a/20. 
Table 4

\begin{tabular}{|c|c|c|}
\hline Name & Gene identity & Function* \\
\hline CMTell & Syntrophin betal, Sntb I & Cytoskeleton component \\
\hline \multirow[t]{4}{*}{ CMTel3 } & Dual specificity protease $22, \mathrm{Dsp} 22$ & Protein tyrosine/serine/threonine phosphatase activity \\
\hline & Interferon regulatory factor 4 , Irf 4 & Transcriptional activator (multiple myeloma oncogene I) \\
\hline & Exocyst complex component 2, Exoc2 & Transport \\
\hline & Hus $/ b$ & Checkpoint protein \\
\hline \multirow[t]{6}{*}{ CMTel4 } & Corin, Corin & Serine protease \\
\hline & Nuclear transcription factor, $\mathrm{X}$-box binding-like, Nfxll & Transcription factor \\
\hline & Cyclic nucleotide gated channel alpha I, Cngal & Potassium ion transport \\
\hline & NIPA-like domain containing I, Npall & Unknown \\
\hline & Tyrosine kinase, $T x k$ & Tyrosine protein kinase, transcription factor \\
\hline & Tyrosine-protein kinease, $\mathrm{Tec}$ & Tyrosine protein kinase, signaling \\
\hline CMSat4 & None & NA \\
\hline
\end{tabular}

*The functionality of these genes has not been verified; table could include pseudogenes and gene fragments. NA, not applicable.

The sequence on the telomeric side of IMFS4 as well as CMTel4 sequences are orthologous to human chromosome 4 p12 and cow chromosome 6. These sequences contain the genes Corin, Nfxl1, Cnga1, Npal1, Txc, and Tec (Tables 3 and 4). Based on the known synteny relationships (see above), we can deduce that CMTel 4 maps to Chinese muntjac chromosome 16 or 21 . Using the same logic as above, IMFS4 thus appears to contain the fusion site between the telomere of an ancestral chromosome related to Chinese muntjac chromosome 16 or 21 and the centromere of an ancestral chromosome related to Chinese muntjac chromosome 21 or 8 . Based on the comparative chromosome map [21] and our FISH studies of IMFS4, there are two potential matching fusion sites on Indian muntjac chromosome $3+X: 16 / 21$ and 21/8.

The sequence on the telomeric side of IMFS5 is orthologous to human chromosome 1p36.13 and cow chromosome 2qtel. Based on the known synteny relationships (see above), we can deduce that the IMFS5 telomeric side is also orthologous to Chinese muntjac chromosome 3 . Using the same logic as above, IMFS 5 thus appears to contain the fusion site between the telomere of an ancestral chromosome related to Chinese muntjac chromosome 3 and the centromere of another ancestral chromosome. Based on the comparative chromosome map [21] and our FISH studies of IMFS5, there are three potential matching fusion sites on Indian muntjac chromosome 1 : 3c/3d, 3b/17, and $3 \mathrm{a} / 20$.

We established that IMFS6 resides on Indian muntjac chromosome 2. The sequence on the telomeric side of IMFS6 is orthologous to human chromosome 10q26.3 and cow chromosome 26 (in a genomic region that contains Cyp2e1; Table 3). Based on the known synteny relationships (see above), we can deduce that the IMFS6 telomeric side is also orthologous to Chinese muntjac chromosome 2. Using the same logic as above, IMFS6 thus appears to contain the fusion site between the telomere of an ancestral chromosome related to Chinese muntjac chromosome 2 and the centromere of another ancestral chromosome. Based on the comparative chromosome map [21] and our FISH studies of IMFS6, there are four potential matching fusion sites on Indian muntjac chromosome 2 : $2 \mathrm{~b} / 2 \mathrm{c}, 2 \mathrm{c} / 2 \mathrm{~d}, 2 \mathrm{~d} / 2 \mathrm{a}$, and $2 \mathrm{a} / 10$.

Finally, the sequence on the telomeric side of IMFS7 is orthologous to human chromosome 4ptel and cow chromosome 6 (in a genomic region that contains Gpi7; Table 3). Based on the known synteny relationships (see above), we can deduce that the IMFS7 telomeric side is also orthologous to Chinese chromosome 16 or 21. Using the same logic as above, IMFS7 thus appears to contain the fusion site between the telomere of an ancestral chromosome related to Chinese muntjac chromosome 16 or 21 and the centromere of an ancestral chromosome related to Chinese muntjac chromosome 21 or 8 . Based on the comparative chromosome map [21] and our FISH studies of IMFS7, there are two potential matching fusion sites on Indian muntjac chromosome $3+\mathrm{X}$ : 16/21 and 21/8.

\section{Discussion}

The strikingly small diploid chromosome number in the Indian muntjac has captured the interest of geneticists for a number of years [2]. The rarity of such a karyotype among mammals suggests that the extant Indian muntjac genome formed through an unusual set of evolutionary events. Here, we applied the tools of comparative genomics to gain clues about that evolutionary history.

Using a BAC-based mapping and sequencing strategy, we isolated, sequenced, and analyzed seven regions of the Indian muntjac genome that appear to reflect ancestral chromosome fusion sites. These genomic regions share a similar organization, containing both specific repeats (telomeric and satellite 


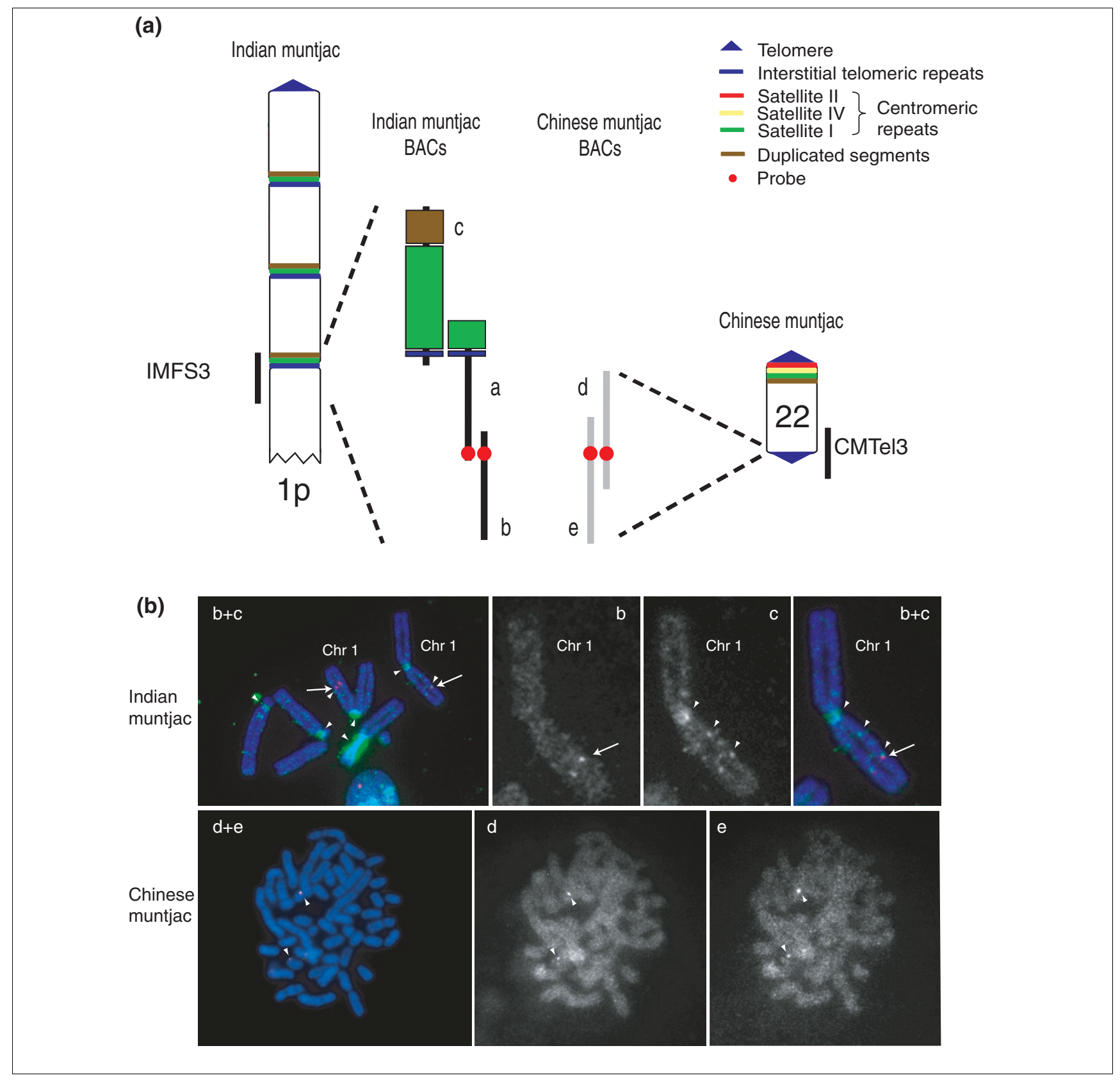

\section{Figure 5}

FISH-based characterization of an ancestral chromosome fusion site in the Indian muntjac genome. (a) Sequence IMFS3 (Table I) was derived from three overlapping Indian muntjac BACs (BACs a-c), and contains the features expected of an ancestral chromosome fusion site (Figure 3). Each of the BACs was mapped by FISH to metaphase chromosomes from Indian muntjac cells. A probe (red circle) derived from the end of the middle clone (BAC a) was used to isolate an overlapping Indian muntjac BAC (BAC b) as well as two orthologous Chinese muntjac BACs (BACs $d$ and e); the latter were sequenced, confirmed to be orthologous to the telomeric end of IMFS3, and designated CMTel3 (Table 2). (b) FISH studies were performed on the five clones (BACs a-e) depicted in (a). Indian muntjac BAC [GenBank:ACI52355] (BAC a) hybridized to an interstitial position on chromosome I and at the neck of chromosome 3+X (shown in Figure Ib). The FISH composite image (upper row, far left) generated by merging the DAPI (blue), Spectrum Orange (red), and Spectrum Green (green) channels shows BAC [GenBank:AC 19764I] (BAC b; in red) hybridizing to an interstitial site on Indian muntjac chromosome I (arrow) and BAC [GenBank:ACl66/88] (BAC c; in green) hybridizing to the latter position as well as other centromeric and interstitial sites (arrowhead), reflecting its high content of satellite I and duplicated segments; an enlarged view of chromosome I (upper row, far right) confirms the site of co-hybridization of these two BACs. The gray scale channel images (upper row, middle) corresponding to the enlarged view of chromosome I show the hybridization pattern of each BAC separately. A similar FISH study with Chinese muntjac chromosomes is shown on the bottom row, with Chinese muntjac BAC [GenBank:AC 96603] (BAC d; in green) and BAC [GenBank:ACI988I5] (BAC e; in red) co-hybridizing to a telomeric position on a pair of chromosomes (arrowheads). The two gray scale channel images show the hybridization pattern of each BAC separately. Synteny analysis suggests that the site of hybridization of these two BACs is Chinese muntjac chromosome 22 (see text). 


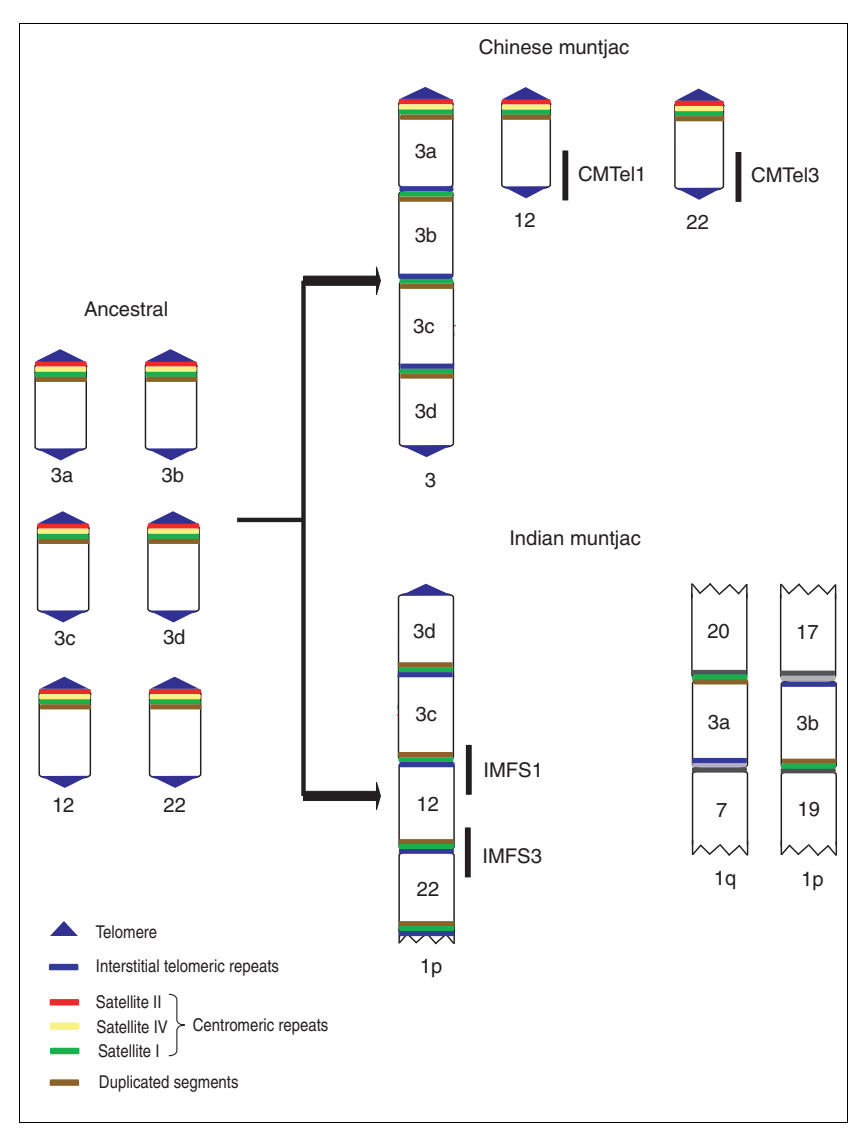

\section{Figure 6}

Evolutionary history of Indian muntjac chromosome fusion sites. A proposed model is shown tracing the evolutionary history of Indian muntjac IMFSI and IMFS3 as well as the orthologous Chinese muntjac sequences CMTell and CMTel3. The hypothetical ancestral muntjac genome contained as many as 70 chromosomes, a small subset of which is shown on the left. There is evidence [18,39] that Chinese muntjac chromosome 3 was derived from three fusion events involving ancestral chromosomes 3a-3d. Ancestral chromosomes 3d, 3c, 12, and 22 appeared to have fused in head-to-tail fusions to form the distal end of Indian muntjac chromosome Ip; note that ancestral chromosomes $3 \mathrm{a}$ and $3 \mathrm{~b}$ fused with other chromosomes and are present elsewhere in the Indian muntjac genome [18,39]. The chromosome fusion sites on Indian muntjac chromosomes contain telomeric repeats adjacent to satellite I sequences (Figures 2 and 3), consistent with sequential head-to-tail fusions of telocentric chromosomes. During these events the telomeric centromere of the 'head' chromosome (containing telomeric repeats and satellite II and IV sequences) becomes lost, and satellite I sequences become fused with telomeric repeats from the 'tail' chromosome.

I) in the expected spatial configuration [19] and duplicated segments (Figures 2 and 3). The architecture of the telomeric side of each ancestral chromosome fusion site is generally less complex than that of the satellite I side. There is clear evidence for breakage of synteny at each site, with the orthology (relative to four other mammalian genomes) on the telomeric side interrupted precisely at the fusion site itself (Figure 4). For the telomeric side of several sites, we further generated orthologous Chinese muntjac sequence, and demonstrated that these sequences emanate from authentic telomeric regions of Chinese muntjac chromosomes (Figure 5).

Our findings provide new details about the chromosome fusion events that led to the Indian muntjac karyotype. With the exception of IMFS7 (which corresponds to TGS400 [19]), the IMFS sequences reported here represent novel, previously unreported Indian muntjac chromosome fusion sites $[19,20]$. Like previously reported fusion sites (TGS40o, TGM225, and TCS165 [19]), the IMFS sequences contain juxtaposed satellite I and telomeric repeats. In all cases, immediately adjacent to satellite I are duplicated genomic segments, a feature that is typical of mammalian pericentromeric regions (Figure 3). This architecture contrasts the observed order of satellite sequences within either the telomeres or centromeres of muntjac chromosomes. For example, in the Formosan muntjac, the order of satellite sequences at the telocentric end of chromosomes is pter - II - IV - I - qter [33]. If this order of centromeric satellites reflects that present in the common ancestral species, the formation of head-to-tail chromosome fusions likely proceeded with a loss of most of the telomeric sequence from the 'tail' chromosome and a loss of telomeric, satellite II, and satellite IV (and potentially some satellite I) sequences from the 'head' chromosome. This would have created new physical associations between pericentromeric duplicated segments and satellite I sequences from the donor 'head' chromosome and telomeric sequences from the donor 'tail' chromosome (Figures 3 and 6). Together with the reported general reduction in intron sizes in Indian muntjac [20], the loss of most of the telomeric repeats and satellites II and IV could at least partially account for the smaller genome of Indian (haploid C-value of $2.1 \mathrm{pg}$ ) versus Chinese (haploid C-values of 2.7) muntjac [7].

Muntjac satellite II is functionally important, having been shown to bind CENPA. The complex of satellite DNA and CENPA marks the site of the active centromere [31]. Multicentric chromosomes can form multiple spindle attachments and have been shown to be lost during cell division. [40-42]. It is thus interesting to note that the loss of satellite II (associated with the chromosome fusion events that occurred during the evolution of the muntjac genome) likely ensured that there were not multiple active centromeres in the resulting Indian muntjac chromosomes.

The main telomeric-repeat block as well as the smaller (TTAGGG) blocks (in IMFS3, IMFS4, and IMFS6) and (TTAGGG) ${ }_{n}$-like blocks (in IMFS1 and IMFS6; Figure 3) represent additional evidence that these regions reflect ancestral telomeres, as such sequences are often found in subtelomeric regions. The presence of small blocks of satellite IV (in IMFS1, IMFS4, IMFS5, and IMFS7) and satellite II (in IMFS4; Figure 3) within the telomeric side of some of these sites suggests that these centromeric satellites may have been present near the telomeres of ancestral muntjac chromosomes (or could 
also suggest that a more complex genomic rearrangement occurred during the chromosome fusion event).

To our knowledge, the current study is the first to reveal the presence of duplicated genomic segments on the satellite I side of muntjac chromosome fusion sites. A duplicated segment orthologous to human chromosome 1q24.1 is present in all of the IMFS sequences reported here except IMFS2 (Figure 4), suggesting that this may have been a commonly duplicated segment within pericentromeric regions of ancestral muntjac chromosomes. Human and rodent pericentromeric regions are known to contain various segmental duplications $[38,43,44]$. Of note, the duplicated segments characterized here (Figures 3 and 4) might be useful as molecular landmarks for identifying additional IMFS sequences in the Indian muntjac genome.

Previous studies have suggested that chromosome fusions are produced by sequence-specific recognition and illegitimate recombination between homologous DNA elements (or other specific motifs) on non-homologous ancestral chromosomes $[14,15,18,19,22,30]$. The repetitive elements identified in the seven IMFS sequences (telomeric repeats, duplicated segments, and satellites I, II, and IV) represent potential candidate targets for such illegitimate recombination events. Exploring the presence and organization of these repetitive elements at orthologous locations in the Chinese muntjac genome would provide additional insights about their involvement in the chromosome fusion events leading to Indian muntjac karyotype.

The general model for chromosome fusion events that involves telomeric repeats and satellite I is supported by the data reported here (Figures 3 and 6), but does not necessarily account for all of the chromosome fusion sites in the Indian muntjac genome. Many more Indian muntjac fusion sites remain to be isolated and characterized; indeed, 29 tandem, head-to-tail fusions would theoretically be required to condense the estimated 70 ancestral chromosomes into the current set of Indian muntjac chromosomes [21]. These additional chromosome fusion sites may differ structurally from the seven described here. Further, a different type of chromosome fusion event (head-to-head, centric fusion) is likely to have yielded the current centromeres of the Indian muntjac chromosomes [12,13,21]. Meanwhile, the Chinese muntjac karyotype appears to have been derived from a common ancestral species through 12 tandem fusions involving 18 chromosomes [22]. Sequencing and characterizing additional chromosome fusion sites in both the Indian and Chinese muntjac genomes would enable a more complete delineation of the evolutionary history of these various fusion events.

It is notable that the chromosome fusion sites we identified in Indian muntjac are architecturally different to the characterized fusion site on human chromosome 2q13 [37] and other regions in the human genome containing interstitial telomeric repeats [26]. The Indian muntjac chromosome fusion sites contain telomeric repeats immediately adjacent to satellite I [19], suggesting that these repetitive sequences played a role in the fusion event. In contrast, most interstitial telomeric sequences in the human genome appear to have been derived from double-strand breakage and repair via nonhomologous end-joining rather than from telomeric fusion events [25]. We isolated and characterized interstitial telomeric sequences in an additional three Indian muntjac BACs ([GenBank:AC154147], [GenBank:AC154148], and [GenBank:AC154923]; data not shown); these clones contain what appears to be short interstitial telomeric sequences similar to those described in humans and other organisms [25,26]. For example, [GenBank:AC154147] has a short block of telomeric repeats flanked by mammalian-wide interspersed repeats/ short interspersed elements (MIR/SINE) that resembles the human class B interstitial telomeric sequences described previously [26]. Note that these BAC sequences were excluded from the main studies reported here because they did not contain closely linked telomeric repeats and satellite I nor was there any evidence of synteny breakage; thus, they are unlikely to represent chromosome fusion sites.

\section{Conclusion}

Our studies help to provide a better understanding of the likely evolutionary history of the Indian muntjac karyotype as well as insights into the paradoxical finding that closely related species can harbor genomes with drastically different organizations. More broadly, the comparative studies of genomes, such as that performed here, are providing insights into how mammalian genomes evolve, why mammals most typically package their genomes into 40-60 chromosomes, and how unusually large (or small) mammalian chromosomes replicate and segregate.

\section{Materials and methods \\ BAC isolation}

BAC clones were isolated from libraries constructed from Indian (CHORI-244) and Chinese (CHORI-245) muntjac [45]; each library was derived from a male individual. The initial isolation of Indian muntjac BACs involved the use of endlabeled telomere repeat-specific oligonucleotide probes [(TTAGGG) $)_{5}$ and (CCCTAA) $)_{5}$. Subsequent isolation of Indian and Chinese muntjac BACs involved the use of 'overgo' probes, which consist of 36-bp double-stranded, radiolabeled DNA molecules generated by performing primer extension with two 22mer oligonucleotides that contain an 8-base complementary region of overlap at their 3 ' ends [46]; overgo probes were designed from the ends of sequenced BACs (see below; probe sequences available on request). 


\section{BAC contig assembly}

Following isolation, BACs were subjected to restriction enzyme digest-based fingerprint analysis [34], allowing their assembly in clone contigs $[47,48]$. In some cases, contigs were extended by isolating overlapping clones using new overgo probes designed from the ends of sequenced BACs.

\section{BAC characterization}

BAC clones were cultured shaking in 2X YT medium (Quality Biological, Gaithersburg, MD, USA) containing $12.5 \mu \mathrm{g} / \mathrm{ml}$ chloramphenicol at $37^{\circ} \mathrm{C}$ overnight, and BAC DNA was purified using an Autogen 740 Automated Plasmid Isolation System (Holliston, MA, USA). For Southern blot analysis, the purified DNA was digested with EcoRI, electrophoretically separated in a $1 \%$ agarose gel, and transferred to Hybond-N membranes (GE Healthcare Bio-sciences, Piscataway, NJ, USA) using standard procedures [49]; membranes were then hybridized, and the resulting images were captured with an FLA-5000 phosphorimager (Fujifilm). Hybridization probes used to analyze the Southern blots included: (TTAGGG) ${ }_{5}$ and $(\text { CCCTAA })_{5}$ telomeric probes (as above); and modified (longer) overgo probes designed from known muntjac satellite-telomeric repeat junctions reported in Hartmann and Scherthan [19]: AGGGTTAGGGTGGAGGCCGCAAATTCAACCTCCCTC and GAGGCTTCTCGCAGCTGTAGGTCTGGTTGAGGGAGG from TGS400 [GenBank:AY322158]; AGGGTTAGGGCATCTCGGGGTCGATTCCAGTGGAGG and ATCTGGGAGAGGGACGTTGAATTTATGGCCTCCACT from TGM225 [GenBank:AY322159]; and ACCCTAACCCTTTGACTGTGTGGATGAAAAGGGGCA and CCCCTGCACTGCGTGCAGAGAAATTCCGTGCCCCTT from TCS165 [GenBank:AY322160]. For FISH studies, the purified BAC DNA was labeled with Spectrum Orange or Spectrum Green d-UTP (Abbott Laboratories, Des Plaines, IL, USA), and hybridized to Indian and Chinese muntjac metaphase spreads [50-52].

\section{Culturing muntjac cell lines}

A fibroblast cell line from a male Indian muntjac (AG15826) was obtained from the Coriell Cell Repositories and cultured in EMEM medium (BioWhittaker, Walkersville, MD, USA) supplemented with $10 \%$ fetal bovine serum, $1 \%$ glutamine, $1 \%$ penicillin-streptomycin, $2 \times$ vitamins (Invitrogen, Carlsbad, CA, USA), and $2 \times$ amino acids (Sigma-Aldrich, St. Louis, MO, USA). A fibroblast cell line from a male Chinese muntjac was kindly provided by Dr BR Brinkley (Baylor College of Medicine) and cultured in Opti-MEM I medium supplemented with $4 \%$ fetal bovine serum, $1 \%$ glutamine, and $1 \%$ penicillinstreptomycin (Invitrogen).

\section{Generation and analysis of muntjac-BAC sequences} Selected BACs were sequenced as part of the NISC Comparative Sequencing Program [53], as described [48]. Following refinement [35], high-quality sequences of overlapping BACs were compiled into a single non-redundant sequence using the program TPF Processor [54]. Repetitive sequences were identified and classified with RepeatMasker (version open3.1.6) [55] using a cow repeat library (Repbase Update 20061006, RM database version 20061006) [56,57].

Known muntjac satellite sequences were identified with NCBI BLAST 2 Sequences $[58,59]$ or RepeatMasker using a custom repeat library compiled for each muntjac species. The Indian muntjac repeat library contained Indian muntjac satellites I [GenBank:X02323], II [GenBank:AF170123], and IV [GenBank:AYo64466]. The Chinese muntjac repeat library contained Chinese muntjac satellites I [GenBank: $\left.\underline{X}_{56823}\right]$ and IV [GenBank:AYo64467] as well as Formosan muntjac satellite II [GenBank:AY380828]. Sequences were annotated for known genes based on matches to human [60-62] (or cow [63], when available) and RefSeq mRNA sequences using Spidey [64]. Multi-species sequence comparisons were performed using MultiPipMaker [65]. Duplicated genomic segments were detected and analyzed using PipMaker [66] and/or LAGAN/VISTA [67].

\section{Synteny analysis}

The generated and assembled Indian muntjac sequences were first analyzed by RepeatMasker (version open-3.1.6) using the cow repeat database (Repbase Update 20061006, RM database version 20061006). The coordinates of satellite and (TTAGGG) $)_{\mathrm{n}}$ tracts were identified, and all sequences matching cow repeats were masked. Each repeat-masked sequence was then used to query (using BLASTN) a database consisting of all Indian muntjac repeat-masked sequences, and non-self matches with $>90 \%$ identity and $>50$ bp in length (reflecting duplicated segments among the sequences) were identified. Similarly, each repeat-masked sequence was used to query (using BLASTN) the assembled genome sequences of human (hg18, NCBI build 36.1) [6o-62], cow (Btau_4.o) [63], dog (canFam2) [68], and mouse (mm9, build 37) [69] that had been downloaded from the UCSC Genome Browser [70]. Human, dog, and mouse sequence matches with a bit score of $>100$ and cow sequence matches with a bit score of $>600$ were displayed. The regions of synteny to the human, cow, dog, and mouse genome-sequence assemblies are shown in Figure 4.

\section{Abbreviations}

BAC: bacterial artificial chromosome; CENPA: centromeric protein A; CMSat: Chinese muntjac satellite; CMTel: Chinese muntjac telomere; FISH: fluorescence in situ hybridization; IMFS: Indian muntjac fusion site; LINE: long interspersed nucleotide element; LTR: long terminal repeat; SINEs: short interspersed nucleotide elements.

\section{Authors' contributions}

VT carried out the laboratory-based studies, performed the primary bioinformatics analyses, and drafted the manuscript. MGS advised on numerous aspects of the study, reviewed the 
results of the bioinformatics analyses, and performed critical reading and editing of the manuscript. $\mathrm{SH}$ and $\mathrm{HR}$ performed the synteny analysis and contributed text and a figure to the manuscript. The NISC Comparative Sequencing Program generated the genomic sequence data. $\mathrm{AD}$ and $\mathrm{EP}$ performed the FISH studies. EDG conceived of the study, participated in its design and coordination, and performed critical editing of all components of the manuscript. All authors read and approved the final manuscript.

\section{Acknowledgements}

We thank the participants of the NISC Comparative Sequencing Program for their generation of high-quality sequence data, in particular Drs Bob Blakesley and Gerry Bouffard, as well as Jyoti Gupta, Shelise Brooks, Pam Thomas, and Betty Benjamin. This work was supported in part by the Intramural Program of the National Human Genome Research Institute, National Institutes of Health.

\section{References}

I. Contreras LC, Torres-Mura JC, Spotorno AE: The largest known chromosome number for a mammal, in a South American desert rodent. Experientia 1990, 46:506-508.

2. Wurster DH, Benirschke K: Indian muntjac, Muntiacus muntjak: a deer with a low diploid chromosome number. Science 1970 , 168: I364-1366.

3. Nakatani $\mathrm{Y}$, Takeda H, Kohara Y, Morishita S: Reconstruction of the vertebrate ancestral genome reveals dynamic genome reorganization in early vertebrates. Genome Res 2007, 17:1254-1265.

4. Hernandez FM, Vrba ES: A complete estimate of the phylogenetic relationships in Ruminantia: a dated species-level supertree of the extant ruminants. Biol Rev Camb Philos Soc 2005, 80:269-302.

5. Lan H, Wang W, Shi L: Phylogeny of Muntiacus (Cervidae) based on mitochondrial DNA restriction maps. Biochem Genet 1995, 33:377-388.

6. Wurster DH, Benirschke K: Chromosome studies in some deer, the springbok, and the pronghorn, with notes on placentation in deer. Cytologia 1967, 32:273-285.

7. Johnston FP, Church RB, Lin CC: Chromosome rearrangement between the Indian muntjac and Chinese muntjac is accompanied by a delection of middle repetitive DNA. Can J Biochem 1982, 60:497-506

8. Liming S, Pathak S: Gametogenesis in a male Indian muntjac $\mathbf{x}$ Chinese muntjac hybrid. Cytogenet Cell Genet I98I, 30:I52-156.

9. Soma H, Kada H, Mtayoshi K, Suzuki Y, Meckvichal C, Mahannop A, Vatanaromya B: The chromosomes of Muntiacus feae. Cytogenet Cell Genet 1983, 35:156-158.

10. Wang W, Lan H: Rapid and parallel chromosomal number reductions in muntjac deer inferred from mitochondrial DNA phylogeny. Mol Biol Evol 2000, 17:1326-1333.

II. Shi LM, Ma CX: A new karyotype of muntjac (Muntiacus sp.) from Gongshan county in China. Zool Res 1988, 9:343-347.

12. Hsu TC, Pathak S, Chen TR: The possibility of latent centromeres and a proposed nomenclature system for total chromosome and whole arm translocation. Cytogenet Cell Genet 1975, I 5:4I-49.

13. Shi L, Ye Y, Duan X: Comparative cytogenetic studies on the red muntjac, Chinese muntjac and their FI hybrids. Cytogenet Cell Genet 1980, 26:22-27.

14. Lin CC, Sasi R, Fan YS, Chen ZQ: New evidence for tandem chromosome fusions in the karyotypic evolution of Asian muntjacs. Chromosoma 1991, 101:19-24.

15. Lee C, Sasi R, Lin CC: Interstitial localization of telomeric DNA sequences in the Indian muntjac chromosomes: further evidence for tandem chromosome fusions in the karyotypic evolution of the Asian muntjacs. Cytogenet Cell Genet 1993, 63:156-159.

16. Yang F, Müller S, Just R, Ferguson-Smith MA, Wienberg J: Comparative chromosome painting in mammals: human and the
Indian muntjac (Muntiacus muntjak vaginalis). Genomics I997, 39:396-40I.

17. Frönicke L, Scherthan $\mathrm{H}$ : Zoo-fluorescence in situ hybridization analysis of human and Indian muntjac karyotypes (Muntiacus muntjak vaginalis) reveals satellite DNA clusters at the margins of conserved syntenic segments. Chromosome Res 1997, 5:254-26I.

18. Yang F, O'Brien PC, Wienberg J, Ferguson-Smith MA: A reappraisal of the tandem fusion theory of karyotype evolution in Indian muntjac using chromosome painting. Chromosome Res 1997 , 5:109-117.

19. Hartmann N, Scherthan H: Characterization of ancestral chromosome fusion points in the Indian muntjac deer. Chromosoma 2004, II 2:213-220.

20. Zhou Q, Huang L, Zhang J, Zhao X, Zhang Q, Song F, Chi J, Yang F, Wang W: Comparative genomic analysis links karyotypic evolution with genomic evolution in the Indian muntjac (Muntiacus muntjak vaginalis). Chromosoma 2006, I I 5:427-436.

21. Chi JX, Huang L, Nie W, Wang J, Su B, Yang F: Defining the orientation of the tandem fusions that occurred during the evolution of Indian muntjac chromosomes by BAC mapping. Chromosoma 2005, II 4:167-172.

22. Yang F, O'Brien PC, Wienberg J, Neitzel H, Lin CC, Ferguson-Smith MA: Chromosomal evolution of the Chinese muntjac (Muntiacus reevesi). Chromosoma 1997, 106:37-43.

23. Huang L, Chi J, Wang J, Nie W, Su W, Yang F: High-density comparative BAC mapping in the black muntjac (Muntiacus crinifrons): molecular cytogenetic dissection of the origin of MCR I +4 in the XIX2Y IY2Y3 sex chromosome system. Genomics 2006, 87:608-615.

24. Huang L, Wang J, Nie W, Su W, Yang F: Tandem chromosome fusions in karyotypic evolution of Muntiacus: evidence from M. feae and M. gongshanensis. Chromosome Res 2006, 14:637-647.

25. Nergadze SG, Rocchi M, Azzalin CM, Mondello C, Giulotto E: Insertion of telomeric repeats at intrachromosomal break sites during primate evolution. Genome Res 2004, I4:1704-1710.

26. Azzalin CM, Nergadze SG, Giulotto E: Human intrachromosomal telomeric-like repeats: sequence organization and mechanisms of origin. Chromosoma 2001, I 10:75-82.

27. Bogenberger J, Schnell H, Fittler F: Characterization of X-chromosome specific satellite DNA of Muntiacus muntjak vaginalis. Chromosoma 1982, 87:9-20.

28. Bogenberger JM, Neumaier PS, Fittler F: The muntjak satellite IA sequence is composed of $3 \mathrm{I}$-base-pair internal repeats that are highly homologous to the $3 \mathrm{I}$-base-pair subrepeats of the bovine satellite I.715. Eur J Biochem 1985, 148:55-59.

29. Lee C, Lin CC: Conservation of a 31 -bp bovine subrepeat in centromeric satellite DNA monomers of Cervus elaphus and other cervid species. Chromosome Res 1996, 4:427-435.

30. Li YC, Lee C, Sanoudou D, Hseu TH, Li SY, Lin CC: Interstitial colocalization of two cervid satellite DNAs involved in the genesis of the Indian muntjac karyotype. Chromosome Res 2000, 8:363-373.

31. Vafa O, Shelby RD, Sullivan KF: CENP-A associated complex satellite DNA in the kinetochore of the Indian muntjac. Chromosoma 1999, 108:367-374.

32. Li YC, Lee C, Chang WS, Li SY, Lin CC: Isolation and identification of a novel satellite DNA family highly conserved in several Cervidae species. Chromosoma 2002, I I I:I76-I83.

33. Lin CC, Chiang PY, Hsieh LJ, Liao SJ, Chao MC, Li YC: Cloning, characterization and physical mapping of three cervid satellite DNA families in the genome of the Formosan muntjac (Muntiacus reevesi micrurus). Cytogenet Genome Res 2004, 105: $100-106$.

34. Marra MA, Kucaba TA, Dietrich NL, Green ED, Brownstein B, Wilson RK, McDonald KM, Hillier LW, McPherson JD, Waterston RH: High throughput fingerprint analysis of large-insert clones. Genome Res 1997, 7:1072-1084.

35. Blakesley RW, Hansen NF, Mullikin JC, Thomas PJ, McDowell JC, Maskeri B, Young AC, Benjamin B, Brooks SY, Coleman BI, Gupta I, Ho SL, Karlins EM, Maduro QL, Stantripop S, Tsurgeon C, Vogt JL, Walker MA, Masiello CA, Guan X, NISC Comparative Sequencing Program, Bouffard GG, Green ED: An intermediate grade of finished genomic sequence suitable for comparative analyses. Genome Res 2004, I 4:2235-2244.

36. Wilson RK, Mardis ER: Shotgun sequencing. In Genome Analysis: A Laboratory Manual Edited by: Birren B, Green E, Klapholz S, Myers R, Roskams J. Cold Spring Harbor, NY: Cold Spring Harbor Laboratory 
Press; 1997:396-454.

37. IJdo JW, Baldini A, Ward DC, Reeders ST, Wells RA: Origin of human chromosome 2: an ancestral telomere-telomere fusion. Proc Natl Acad Sci USA 199I, 88:905I-9055.

38. Bailey JA, Yavor AM, Massa HF, Trask BJ, Eichler EE: Segmental duplications: organization and impact within the current human genome project assembly. Genome Res 200I, II:1005-1017.

39. Chi J, Fu B, Nie W, Wang J, Graphodatsky AS, Yang F: New insights into the karyotypic relationships of Chinese muntjac (Muntiacus reevesi), forest musk deer (Moschus berezovskii) and gayal (Bos frontalis). Cytogenet Genome Res 2005, 108:310-316.

40. Koshland D, Hartwell LH: The structure of sister minichromosome DNA before anaphase in Saccharomyces cerevisiae. Science 1987, 238: $1713-1716$.

41. Lukaszewski AJ: Chromatid and chromosome type breakagefusion-bridge cycles in wheat (Triticum aestivum L.). Genetics 1995, 140:1069-1085.

42. McClintock $B$ : The behavior in successive nuclear divisions of a chromosome broken at meiosis. Proc Natl Acad Sci USA 1939, 25:405-416.

43. Thomas JW, Schueler MG, Summers TJ, Blakesley RW, McDowell JC, Thomas PJ, Idol JR, Maduro VV, Lee-Lin SQ, Touchman JW, Bouffard GG, Beckstrom-Sternberg SM, NISC Comparative Sequencing Program, Green ED: Pericentromeric duplications in the laboratory mouse. Genome Res 2003, 13:55-63.

44. Tuzun E, Bailey JA, Eichler EE: Recent segmental duplications in the working draft assembly of the brown Norway rat. Genome Res 2004, 14:493-506.

45. BACPAC Resources Center [http://bacpac.chori.org/]

46. Vollrath D: DNA markers for physical mapping. In Genome Analysis: A Laboratory manual Edited by: Birren B, Green E, Klapholz S, Myers R, Roskams J. Cold Spring Harbor, NY: Cold Spring Harbor Laboratory Press; 1999:187-216.

47. Thomas JW, Prasad AB, Summers TJ, Lee-Lin SQ, Maduro VV, Idol JR, Ryan JF, Thomas PJ, McDowell JC, Green ED: Parallel construction of orthologous sequence-ready clone contig maps in multiple species. Genome Res 2002, I 2: I277-I285.

48. Thomas JW, Touchman JW, Blakesley RW, Bouffard GG, BeckstromSternberg SM, Margulies EH, Blanchette M, Siepel AC, Thomas PJ, McDowell JC, Maskeri B, Hansen NF, Schwartz MS, Weber RJ, Kent W], Karolchik D, Bruen TC, Bevan R, Cutler DJ, Schwartz S, Elnitski L, Idol JR, Prasad AB, Lee-Lin SQ, Maduro VV, Summers TJ, Portnoy ME, Dietrich NL, Akhter N, Ayele K, et al:: Comparative analyses of multi-species sequences from targeted genomic regions. Nature 2003, 424:788-793.

49. Sambrook J, Fritsch EF, Maniatis T: Molecular Cloning: A Laboratory Manual Cold Spring Harbor, NY: Cold Spring Harbor Laboratory Press; 1989.

50. Dutra AS, Mignot E, Puck JM: Gene localization and syntenic mapping by FISH in the dog. Cytogenet Cell Genet 1996, 74:113-117.

51. Lichter P, Cremer T, Borden J, Manuelidis L, Ward DC: Delineation of individual human chromosomes in metaphase and interphase cells by in situ suppression hybridization using recombinant DNA libraries. Hum Genet 1988, 80:224-234.

52. Pinkel D, Straume T, Gray JW: Cytogenetic analysis using quantitative, high-sensitivity, fluorescence hybridization. Proc Natl Acad Sci USA 1986, 83:2934-2938.

53. NISC Comparative Sequencing Program [http:// www.nisc.nih.gov/]

54. TPF Processor [http://www.ncbi.nlm.nih.gov/projects/zoo_seq/]

55. RepeatMasker [http://www.repeatmasker.org/]

56. Jurka J, Kapitonov VV, Pavlicek A, Klonowski P, Kohany O, Walichiewicz J: Repbase Update, a database of eukaryotic repetitive elements. Cytogenet Genome Res 2005, I I 0:462-467.

57. Repbase Update [http://www.girinst.org/Repbase Update.html]

58. Tatusova TA, Madden TL: BLAST 2 Sequences, a new tool for comparing protein and nucleotide sequences. FEMS Microbiol Lett 1999, I 74:247-250.

59. BLAST 2 Sequences [http://www.ncbi.nlm.nih.gov/blast/b/2seq/ wblast2.cgi]

60. International Human Genome Sequencing Consortium: Initial sequencing and analysis of the human genome. Nature 200I, 409:860-921.

6I. Venter JC, Adams MD, Myers EW, Li PW, Mural RJ, Sutton GG, Smith HO, Yandell M, Evans CA, Holt RA, Gocayne JD, Amanatides P, Ballew RM, Huson DH, Wortman JR, Zhang Q, Kodira CD, Zheng
XH, Chen L, Skupski M, Subramanian G, Thomas PD, Zhang J, Gabor Miklos GL, Nelson C, Broder S, Clark AG, Nadeau J, McKusick VA, Zinder $\mathrm{N}$, et al.: The sequence of the human genome. Science 200I, $291:|304-135|$.

62. International Human Genome Sequencing Consortium: Finishing the euchromatic sequence of the human genome. Nature 2004, 43 I:93I-945.

63. Bovine Genome Project [http://www.hgsc.bcm.tmc.edu/ projects/bovine/]

64. Spidey [http://www.ncbi.nlm.nih.gov/IEB/Research/Ostell/Spidey/]

65. Schwartz S, Elnitski L, Li M, Weirauch M, Riemer C, Smit A, NISC Comparative Sequencing Program, Green ED, Hardison RC, Miller W: MultiPipMaker and supporting tools: Alignments and analysis of multiple genomic DNA sequences. Nucleic Acids Res 2003, 31:35 I 8-3524.

66. Schwartz S, Zhang Z, Frazer KA, Smit A, Riemer C, Bouck J, Gibbs R, Hardison R, Miller W: PipMaker - a web server for aligning two genomic DNA sequences. Genome Res 2000, 10:577-586.

67. Brudno M, Do CB, Cooper GM, Kim MF, Davydov E, NISC Comparative Sequencing Program, Green ED, Sidow A, Batzoglou S: LAGAN and Multi-LAGAN: efficient tools for large-scale multiple alignment of genomic DNA. Genome Res 2003, |3:72|-73|.

68. Lindblad-Toh K, Wade CM, Mikkelsen TS, Karlsson EK, Jaffe DB, Kamal M, Clamp M, Chang JL, Kulbokas EJ 3rd, Zody MC, Mauceli E, Xie X, Breen M, Wayne RK, Ostrander EA, Ponting CP, Galibert F, Smith DR, Dejong PJ, Kirkness E, Alvarez P, Biagi T, Brockman W, Butler J, Chin CW, Cook A, Cuff J, Daly MJ, DeCaprio D, Gnerre S: Genome sequence, comparative analysis and haplotype structure of the domestic dog. Nature 2005, 438:803-819.

69. Mouse Genome Sequencing Consortium: Initial sequencing and comparative analysis of the mouse genome. Nature 2002 420:520-562.

70. UCSC Genome Browser: assembled genome sequences [ftp://hgdownload.cse.ucsc.edu/goldenPath]

7I. UCSC Genome Browser [http://genome.ucsc.edu/] 